\title{
Spin-Dependent Conductance in Nonmagnetic InGaAs Asymmetric Double Barrier Devices
}

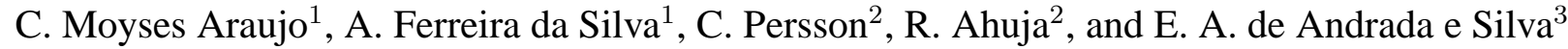 \\ ${ }^{1}$ Instituto de Física, Universidade Federal da Bahia, 40210-340 Salvador, Bahia, Brazil \\ ${ }^{2}$ Department of Physics, Uppsala University, P.O. Box 530, SE 75121 Uppsala, Sweden \\ ${ }^{3}$ Instituto Nacional de Pesquisas Espaciais, CP515, São Jose dos Campos, São Paulo, Brazil
}

Received on 31 March, 2003

\begin{abstract}
The spin dependence of the conductance of an asymmetric double-barrier InGaAs device is studied within the multiband $\mathrm{k} \cdot \mathrm{p}$ and envelope function approximations. The spin-dependent transmission probability for electrons across the structure is obtained using transfer matrices and the low bias conductance per unit area is calculated as a function of the Fermi energy (or doping) in the contacts. The possibility to obtain spin polarized currents in such devices is demonstrated, however, the resulting degree of polarization is rather small (a few percent) in the specific InGaAs structures considered here.
\end{abstract}

\section{Introduction}

The spin-orbit interaction allows the spin manipulation in nonmagnetic semiconductor devices. Besides the spin transistor proposed by Data and Das [1], which is based on the Rashba spin-orbit term, of importance to the spintronics development, there have been, more recently, different proposals of nonmagnetic semiconductor spin filter devices $[2,3,4]$. However, in order to fabricate them, further investigations are necessary in order to test these ideas in different structures and device geometries.

The physical effect behind these electron spin filtering devices is the resonant tunneling through spin-orbit split resonances. The fabrication of the devices for such resonant tunneling experiments includes the creation of mesas in the multibarrier semiconductor structure and subsequent etching, so that the back contact can be made in a doped epilayer near the substrate $[4,5]$. In this case, the electric current will run as schematically illustrated in Fig. 1. As first suggested by Koga et al. [4], a spin-dependent electric current is expected to be detected if the back contact is placed in only one of the sides, with opposite polarizations for right and left sided contacts.

A triple barrier structure with modulation doping was used in Ref. [4] to demonstrate the possibility of getting spin polarized currents in these resonant tunneling devices. In the present contribution, we consider instead structures with undoped asymmetric InGaAs double barriers, and calculate the low bias conductance as a function of the Fermi energy (or doping) in the contacts. The results show that spin dependent electric currents are expected to run in such devices, but with a small polarization degree. Next, we describe the model calculation, present specific results for $\mathrm{InGaAs} / \mathrm{InP} /$ InGaAs/ GaAsSb/InGaAs asymmetric double barrier structures and then summarize the main conclusions.

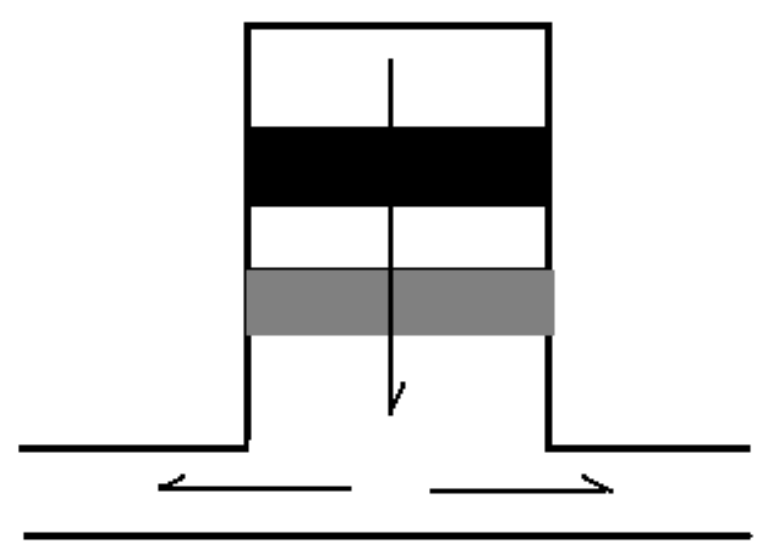

Figure 1. Schematic illustration of the resonant tunneling device structure and geometry, with an asymmetric double barrier and a sided back gate contact.

\section{Spin-dependent conductance}

Let us consider a device geometry as illustrated in Fig. 1. The active part is the InGaAs asymmetric double barrier; and the emitter (front) and collector (back) contacts are assumed to be located at the top and at one of the sides below, respectively, so that the current will flow as illustrated. All interfaces are assumed free from imperfections, so that electrons tunnel with conserved $k_{\|}$(the wave vector parallel to the interfaces) and the sided back contact collects only those electrons with $k_{\|}$different than zero and corresponding to electrons going to the right or to the left. Due to the Rashba effect in this inverse asymmetric structure, the electric current running to the right and to the left will present opposite spin polarizations. Here we calculate such polarization as a function of the Fermi energy in the contacts, in the small bias regime. 
Table I. Band and structure parameters used in the calculation.

\begin{tabular}{ccccc}
\hline & $E_{g}(\mathrm{eV})$ & $\Delta(\mathrm{eV})$ & $m_{0}^{*}\left(m_{0}\right)$ & $V_{c}(\mathrm{meV})$ \\
\hline$I n_{0.53} G a_{0.47} A s$ & 0.75 & 0.36 & 0.04 & 0 \\
$\operatorname{InP}$ & 1.42 & 0.11 & 0.08 & 180 \\
$G a A s_{0.5} S b_{0.5}$ & 0.81 & 0.75 & 0.04 & 360 \\
\hline
\end{tabular}

At zero temperature, the corresponding low bias spin dependent conductance per unit area is given simply as [6]:

$$
G_{\alpha}=\frac{e^{2} m\left(E_{F}\right) E_{F}}{4 \pi^{2} \hbar^{2}} \int_{0}^{\pi / 2} d \theta \sin (\theta) \cos (\theta) T_{\alpha}\left(E_{F}, \theta\right)
$$

where $E_{F}$ is the Fermi energy at the doped InGaAs contact layers, $\theta$ corresponds to the electron angle of incidence, (in the sum over $\left.k_{\|}\right), e$ is the electric charge, $m\left(E_{F}\right)$ is the electron effective mass (obtained from eight band Kane model) at the Fermi energy and $T_{\alpha}$ is the spin dependent transmission probability ( $\alpha=$ up or down, with respect to the direction perpendicular to both the growth and the sided back contact directions). The integral is done numerically and it is important to note that the integration over $\theta$, between zero and $\pi / 2$, assures that we are counting only the electrons in the bottom contact that reach the gate positioned on the right side.

Finally, $T_{\alpha}(E, \theta)$ is calculated following the transfer matrix procedure described in reference [7]. It is based on Kane's model for the bulk plus spin-dependent boundary conditions for the envelope functions [8].

\section{Results}

In Fig. 2, we show the obtained conductance, in units of $e^{2} / h a^{2}$, where $a$ stands for the effective Bohr radius, for both spin up and down electrons. We have considered $3 \mathrm{~nm}$ InP and GaAsSb barriers with a $20 \mathrm{~nm}$ InGaAs layer in between (see Fig. 1). In Table 1, we list the band parameters used in the calculations, i.e. the band gap $E_{g}$, the spin-orbit splitting in valence band $\Delta$, the conduction band edge effective mass and the conduction band offset $V_{c}$, of the different lattice-matched III-V compounds.

One can see that the conductance in such devices, for both spin directions, shows typical steps as a function of the Fermi energy, corresponding to the quantized resonant states (the bottom of the subbands of quasi-bound states). With increasing Fermi energy, the steps in the conductance increase due to the increased transmission probability through the higher subbands. More interesting however, is the small difference seen between the current for opposite spins, which increases with $E_{F}$. This is the signature of the Rashba effect and the expected polarization effect, similar to the one discussed in Ref. [4].

In the inset of Fig. 2, we show the obtained polarization calculated as $P=\left(G_{\text {up }}-G_{\text {down }}\right) /\left(G_{\text {up }}+G_{\text {down }}\right)$. The polarization is seen to be always rather small (only a few percent) and to oscillate as a function of $E_{F}$, due to the polarization inversion at the incoming of each new subband.

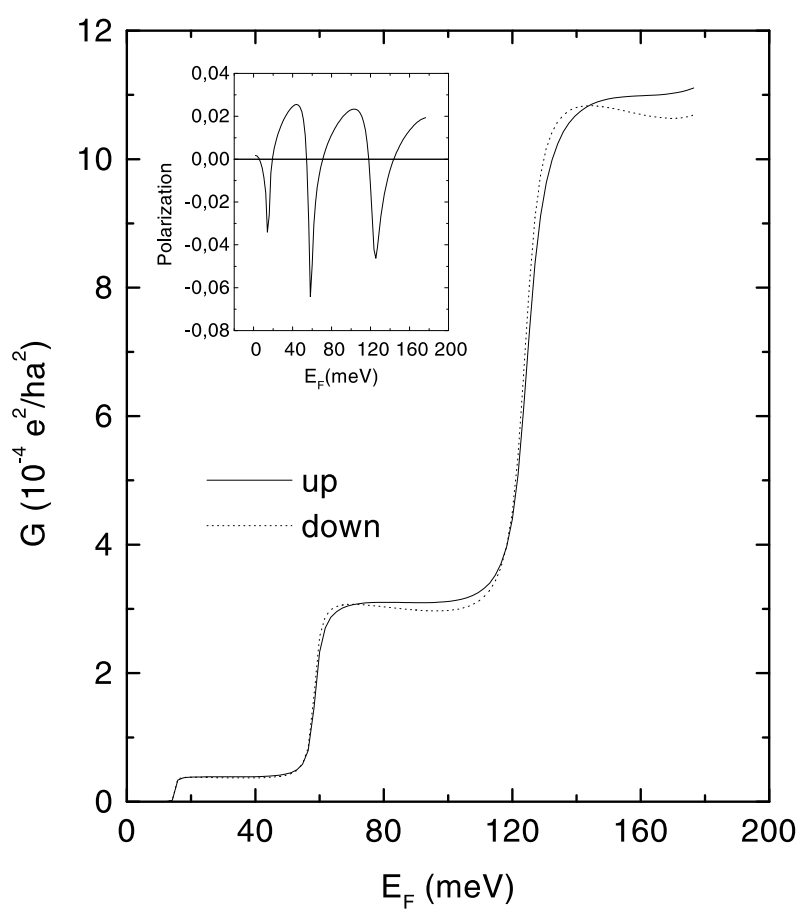

Figure 2. Obtained spin-dependent conductance, per unit area, of the device in Fig. 1, as a function of the Fermi energy (or doping) in the contacts. The band parameters used in calculation are listed in Table 1. The inset shows the corresponding polarization, i.e. $P=\left(G_{u p}-G_{\text {down }}\right) /\left(G_{u p}+G_{\text {down }}\right)$.

\section{Conclusion}

Summarizing, we have studied the spin dependent conductance through InGaAs double barrier structures with sided gated contacts. Our results demonstrate that it is possible to obtain spin polarized electric currents through such nonmagnetic resonant tunneling devices. The predicted polarization, however, is of only a few percent. With a different, probably more sophisticated, device, we may be able to increase considerably such electric current spin polarizaztion. For example, it would be interesting to investigate similar effects in a resonant tunneling transistor, with an asymmetric superlattice [7] in the base, and high energy injection [9]. This work is in progress.

\section{Acknowledgments}

This research was supported by CAPES, CNPq and FAPESP. 


\section{References}

[1] S. Datta and B. Das, Appl. Phys. Lett. 56, 665 (1990).

[2] E. A. de Andrada e Silva and G. C. La Rocca, Phys. Rev. B 59, R15583 (1999).

[3] A. Voskoboynikov, S. Shin Liu and C.P. Lee, Phys. Rev. B 59, 12514 (1999).

[4] T. Koga et al., Phys. Rev. Lett. 88, 126601 (2002).

[5] G. Reuscher et al.,Phys. Rev. B 53, 16414 (1996).
[6] See, for example, J. H. Davies, The physics of lowdimensional semiconductors (Cambridge Univ. Press, Cambridge MA, 1998).

[7] C. Moyses Araujo, A. Ferreira da Silva and E. A. de Andrada e Silva, Phys. Rev. B 65, 235305 (2002).

[8] E. A. de Andrada e Silva, G.C. La Rocca and F. Bassani, Phys. Rev. B 55 , 16293 (1997).

[9] F. Capasso and R. A. Kiehl, J. Appl. Phys. 58, 1366 (1985). 